\title{
Layer-specific speckle tracking analysis of left ventricular systolic function and synchrony in maintenance hemodialysis patients
}

Chang Liu* ${ }^{*}$, Zi-Ning Yan, Li Fan, Jun Huang, Dan Shen and Xiang-Ting Song

\begin{abstract}
Background: This study investigated the value of layer-specific strain analysis by two-dimensional speckle tracking echocardiography (2D-STE) for evaluating left ventricular (LV) systolic function and synchrony in maintenance hemodialysis (MHD) patients.

Methods: A total of 34 MHD patients and 35 healthy controls were enrolled in this study. Dynamic images were collected at the LV apical long-axis, the four- and two- chamber, and the LV short-axis views at the basal, middle, and apical segments. The layer-specific speckle tracking (LST) technique was used to analyze the longitudinal strain (LS) and circumferential strain (CS) of LV sub-endocardium, mid-myocardium, sub-epicardium, global longitudinal strain (GLS), global circumferential strain (GCS), the LV 17 segment time to peak LS (TTP), and the peak strain dispersion (PSD). The differences in these parameters were compared between control and MHD groups, and the correlation between PSD and each LS parameter was examined. The receiver operator characteristic (ROC) curve was used to evaluate the efficacy of three myocardial layer LS and CS in the assessment of LV systolic dysfunction in MHD patients.
\end{abstract}

Results: MHD patients had comparable left ventricular ejection fraction (LVEF), but significantly smaller LV GLS, GCS, and three-layer LS and CS compared to the control group. The three myocardial layer LS of the basal segment, middle segment, and apex segment was significantly reduced in the MHD patients compared to the normal subjects, while the three myocardial layer CS of the basal segment, middle segment, and apex segment was significantly reduced in the MHD patients compared to the normal subjects, except for the sub-endocardium of the middle and apex segment. MHD patients had significantly higher TTP of LV 17 segments and PSD compared to controls, and had delayed peak time in most segments. In addition, PSD of MHD patients was positively correlated with sub-endocardial and mid-myocardial LS and GLS, but not with sub-epicardial LS. The area under the curves (AUCs) of sub-endocardial, mid-myocardial, and sub-epicardial LS in MHD patients were 0.894, 0.852, and 0.870, respectively; the AUCs of sub-epicardial, mid-myocardial, and sub-endocardial CS were 0.852, 0.837, and 0.669, respectively.

Conclusions: LST may detect early changes of all three-layer LS and CS and PSD in MHD patients, and is therefore a valuable tool to diagnose LV systolic dysfunction in MHD patients.

Keywords: Echocardiography, Maintenance hemodialysis, Ventricular function, Synchronicity, Layer-specific strain

\footnotetext{
* Correspondence: 524341735@qq.com

Department of Echocardiography, The Affiliated Changzhou No.2 People's

Hospital of Nanjing Medical University, Changzhou 213003, China
}

(c) The Author(s). 2020 Open Access This article is distributed under the terms of the Creative Commons Attribution 4.0 International License (http://creativecommons.org/licenses/by/4.0/), which permits unrestricted use, distribution, and reproduction in any medium, provided you give appropriate credit to the original author(s) and the source, provide a link to the Creative Commons license, and indicate if changes were made. The Creative Commons Public Domain Dedication waiver (http://creativecommons.org/publicdomain/zero/1.0/) applies to the data made available in this article, unless otherwise stated. 


\section{Background}

End stage renal disease (ESRD) is the final manifestation of chronic renal insufficiency. Hemodialysis, which removes blood metabolites, reduces sodium and water retention, and maintains electrolytes and acid-base balance through solute exchange, is the mainstay treatment for ESRD patients who cannot undergo kidney transplantation [1]. Long-term hemodialysis (i.e. maintenance hemodialysis, MHD) has been proven to be effective in reducing clinical symptoms and improving the quality of life of ESRD patients. However, despite the advances in hemodialysis technology, the mortality and morbidity of ESRD patients on MHD remains high and the quality of life of these patients is poor [2]. Cardiovascular disease is a major complication of ESRD that is caused by metabolic and hemodynamic changes due to ESRD and is associated with high mortality [3, 4], accounting for approximately $50 \%$ of hemodialysis patient deaths [5]. Left ventricular (LV) dysfunction is a critical indicator of cardiac dysfunction and an early clinical manifestation of LV hypertrophy. Thus, LV dysfunction holds important prognostic value for mortality of ESRD patients on MHD [6, 7]. There is a significant need to employ effective screening methods to identify early changes in cardiac function in ESRD patients on MHD.

Two-dimensional speckle tracking echocardiography (2D-STE), which can non-invasively, semi-automatically, and quantitatively analyze ventricular myocardial strain function, has been widely used in the clinic to evaluate cardiac function $[8,9]$. Compared with traditional echocardiography, 2D-STE technology is faster, more accurate, and angle-independent; it can assess myocardial function through longitudinal, circumferential, radial, and torsional motions [10], and thus can examine the global and local left ventricular function with high sensitivity. The layerspecific speckle tracking (LST) technology, derived from 2D-STE, can analyze strain parameters of LV function in three muscular layers - the sub-endocardium, midmyocardium, and sub-epicardium [11]. Therefore, LST allows early detection of subtle ventricular systolic dysfunction [11-13]. Previous studies used the LST to assess global longitudinal, circumferential, and radial myocardial strains of dialysis patients [14-16], but few studies have been performed to measure longitudinal and circumferential strains of the LV three-layer myocardium. In addition, LV systolic synchrony is a sensitive parameter that may reflect early cardiac dysfunction. A normal heart has a well-synchronized LV systolic function, and dyssynchrony is observed in stress, such as in response to pharmacological stimulation [17]. However, using LST to evaluate LV systolic synchrony in MHD patients has been rarely reported.

In the present study, we used LST to evaluate the longitudinal and circumferential strains of the three-layer
LV muscles in MHD patients and to study LV systolic synchrony, with the aim of evaluating the value of LST in detecting cardiac dysfunction at an early stage of MHD.

\section{Methods \\ Ethics statement}

This study protocol was approved by the Ethics Committee of Changzhou Second People's Hospital affiliated to Nanjing Medical University, and all participants signed consent.

\section{Participant selection}

A total of 34 ESRD patients on MHD who were admitted to the Changzhou Second People's Hospital affiliated with Nanjing Medical University between September 2018 and June 2019 and 35 sex- and age-matched healthy individuals (the control group) were registered in this study. Patients who had all of the following were included in this study: 1) kidney disease as the primary disease; 2) LV ejection fraction (LVEF) $\geq 50 \%$; 3) all MHD patients were treated with hemodialysis through forearm arteriovenous anastomosis; 4) hemodialysis was performed three times per week, $4 \mathrm{~h}$ each time; 5) hemodialysis lasted 10-36 months; and 6) each patient was weighed before and after hemodialysis, and the post-dialysis weight was equal to the ideal dry weight of each patient (clinically determined), and the difference in weight before and after dialysis was equal to the total volume of hemodialysis removed. All patients underwent image acquisition and measurement within $30 \mathrm{~min}$ after hemodialysis. Patients who had one of the following diseases were excluded from this study: congenital heart disease, valvular heart disease, coronary artery disease, cardiomyopathy and other heart diseases, arrhythmia, and a history of pulmonary hypertension. Control subjects had normal physical examinations, electrocardiograms, x-ray examinations, and echocardiography results, and did not have any of the following diseases: hypertension, diabetes, heart disease, or abnormal liver and kidney function.

\section{Data collection}

The demographic and clinical data of all participants were obtained from interviews and the hospital database.

\section{Conventional 2D Doppler echocardiography}

Thirty-four MHD patients and 35 control subjects underwent conventional 2D Doppler echocardiography (Vivid E9, GE). The M5S probe was used at a frequency of $2-4.5 \mathrm{MHz}$, and $\mathrm{M}$-mode was used to measure the left ventricular internal diameter at end-diastole (LVIDD), left ventricular internal diameter at endsystole (LVIDS), interventricular septal thickness at end- 
Table 1 Comparison of demographic and clinical characteristics between control and MHD groups

\begin{tabular}{|c|c|c|c|}
\hline & $\begin{array}{l}\text { MHD } \\
(n=34)\end{array}$ & $\begin{array}{l}\text { Control } \\
(n=35)\end{array}$ & $P$-value \\
\hline Age (years) & $52 \pm 9$ & $49 \pm 10$ & 0.327 \\
\hline Male gender (\%) & 52.9 & 48.6 & \\
\hline HD time (months) & $23(10-36)$ & & \\
\hline Heart rate (beats/min) & $76.79 \pm 9.63$ & $\begin{array}{l}72.34 \pm \\
10.50\end{array}$ & 0.071 \\
\hline BMl & $22.18 \pm 2.11$ & $\begin{array}{l}21.29 \pm \\
2.14\end{array}$ & 0.086 \\
\hline Remove volume (kg) & $1.82 \pm 0.93$ & & \\
\hline Dry weight (kg) & $52.07 \pm 8.95$ & & \\
\hline $\mathrm{SBP}(\mathrm{mmHg})$ & $\begin{array}{l}144.65 \pm \\
17.00\end{array}$ & $\begin{array}{l}121.40 \pm \\
6.49\end{array}$ & $<0.001$ \\
\hline $\mathrm{DBP}(\mathrm{mmHg})$ & $88.82 \pm 11.17$ & $\begin{array}{l}75.91 \pm \\
6.29\end{array}$ & $<0.001$ \\
\hline Creatinine (umol/L) & $\begin{array}{l}855.41 \pm \\
191.36\end{array}$ & $\begin{array}{l}55.86 \pm \\
9.92\end{array}$ & $<0.001$ \\
\hline \multicolumn{4}{|l|}{ Cause of ESRD } \\
\hline $\begin{array}{l}\text { Glomerulonephritis } \\
\text { (cases) }\end{array}$ & $19(55.8 \%)$ & & \\
\hline $\begin{array}{l}\text { Diabetic Nephropathy } \\
\text { (cases) }\end{array}$ & $6(17.6 \%)$ & & \\
\hline $\begin{array}{l}\text { Hypertensive nephrosclerosis } \\
\text { (cases) }\end{array}$ & $5(14.7 \%)$ & & \\
\hline Polycystic kidney (cases) & $4(11.8 \%)$ & & \\
\hline
\end{tabular}

Data are expressed as mean \pm SD. Bold number: $P<0.05$

$H D$ hemodialysis; $B M I$ body mass index; $B P$ systolic blood pressure; $D B P$ diastolic blood pressure; Creatinine: serum creatinine; ESRD end-stage renal disease

diastole (IVST), left ventricular posterior wall thickness at end-diastole (LVPWT), left atrial diameter (LAD), and LVEF. Each parameter was measured three times and the average was used to determine the left ventricular mass index (LVMI) using the following formula: LVMI = left ventricular mass / body surface area; left ventricular mass $(\mathrm{LVM})=0.8 \times 1.04 \times[($ LVIDD + LVPWT + IVST $)^{3}-$ LVIDD $\left.^{3}\right]+0.6$; body surface area $($ BSA $)=0.0061$ $\mathrm{x}$ height $+0.0128 \mathrm{x}$ body weight -0.1529 .

Subjects were situated in the left lateral position, connected to the electrocardiogram, and requested to hold their breath to ensure image quality. The instrument was adjusted to clearly display the endocardium, with the image frame rate of 60-90 frames/s. The following images were collected during three consecutive cardiac cycles: LV apical long-axis, two- and four-chamber, and LV short-axis views of the basal, middle, and apical segments. All images were stored in the hard drive.

\section{Data analysis of LV systolic function}

Dynamic images of the basal, middle, and apical segments obtained at the LV short-axis view, and dynamic images of the long-axis, two- and four- chamber views obtained at the LV apical view were imported into the 2D speckle tracking analysis software (2D-Strain, EchoPac. PC version 201, GE Healthcare, Horten, Norway) for analysis. Briefly, the LV endocardium in the apical long-axis chamber view was first delineated, followed by the automatic depiction of the region of interest (ROI), including the LV sub-endocardial, mid-myocardium, and sub-epicardial. Then, the curves of the endocardium and epicardium were manually adjusted to match the LV wall. The system automatically selected the aortic valve closing time point according to the ECG, and automatically generated the strain curves and values of each segment.

Using a similar method, the apical four- and twochamber heart images were analyzed to obtain the subendocardial, mid-myocardial, and sub-epicardial longitudinal strain (LS) of LV 17 segments and their corresponding curves, as well as the bull's eye diagram and to calculate the LV three-layer LS. The LV global longitudinal strain (GLS) is equivalent to the mean LS of the three layers of myocardium. The system also automatically generated LV 17 segment time to peak LS (TTP) and peak strain dispersion (PSD). Three dynamic images of the LV short axis were traced clockwise from the anterior septum, and the software automatically generated ROI including sub-endocardial, mid-myocardial, and sub-epicardial myocardium. If automatic tracking did not yield satisfactory outcomes, manual adjustment was then performed. The system automatically generated the myocardial circumferential strain (CS) of 17 segments and corresponding curves, and calculated the LV threelayer myocardial CS, the CS of the basal, middle, and apical segments. The LV global circumferential strain (GCS) is equivalent to the mean CS of the three layers of myocardium.

Table 2 Comparison of traditional echocardiography parameters between control and MHD groups

\begin{tabular}{llll}
\hline & MHD & Normal & P-value \\
\hline LVIDD $(\mathrm{mm})$ & $52.56 \pm 6.13$ & $46.06 \pm 3.07$ & $<\mathbf{0 . 0 0 1}$ \\
LVIDS $(\mathrm{mm})$ & $36.65 \pm 5.55$ & $31.11 \pm 2.84$ & $<\mathbf{0 . 0 0 1}$ \\
IVST $(\mathrm{mm})$ & $11.56 \pm 1.62$ & $8.86 \pm 1.38$ & $<\mathbf{0 . 0 0 1}$ \\
LVPWT $(\mathrm{mm})$ & $11.24 \pm 1.50$ & $8.14 \pm 1.38$ & $<\mathbf{0 . 0 0 1}$ \\
LAD $(\mathrm{mm})$ & $42.38 \pm 5.29$ & $34.86 \pm 3.12$ & $<\mathbf{0 . 0 0 1}$ \\
LVEF $(\%)$ & $58.24 \pm 5.16$ & $60.11 \pm 4.00$ & 0.095 \\
LVMI $\left(\mathrm{g} / \mathrm{m}^{2}\right)$ & $56.59 \pm 9.11$ & $31.60 \pm 3.82$ & $<\mathbf{0 . 0 0 1}$
\end{tabular}

Data are expressed as mean \pm SD. Bold number: $P<0.05$

LVIDD left ventricular internal diameter at end-diastole; LVIDS left ventricular internal diameter at end-systole; IVST interventricular septal thickness at enddiastole; $L V P W T$ left ventricular posterior wall thickness at end-diastole; $L A D$ left atrial diameter; $L V E F$ left ventricular ejection fraction; $L V M I$ left ventricular mass index 
Table 3 Comparison of global and layer-specific LS and CS between control and MHD groups

\begin{tabular}{|c|c|c|c|c|c|c|c|c|}
\hline \multirow[t]{2}{*}{ Varible } & \multicolumn{4}{|c|}{ Longitudinal strain (\%) } & \multicolumn{4}{|c|}{ Circumferential strain(\%) } \\
\hline & Sub-endocardial & Mid-myocardial & Sub-epicardial & Global & Sub-endocardial & Mid-myocardial & Subepicardial & Global \\
\hline MHD & $-17.04 \pm 4.09$ & $-14.76 \pm 4.11$ & $-13.50 \pm 2.99$ & $-15.07 \pm 3.46$ & $-24.70 \pm 4.68$ & $-15.33 \pm 2.44$ & $-9.20 \pm 1.67$ & $-16.41 \pm 2.59$ \\
\hline Normal & $-23.41 \pm 3.58$ & $-20.18 \pm 3.25$ & $-18.07 \pm 2.75$ & $-20.55 \pm 3.16$ & $-27.40 \pm 3.51$ & $-18.94 \pm 2.74$ & $-12.36 \pm 2.52$ & $-19.57 \pm 2.75$ \\
\hline$P$-value & $<0.001$ & $<0.001$ & $<0.001$ & $<0.001$ & 0.008 & $<0.001$ & $<0.001$ & $<0.001$ \\
\hline
\end{tabular}

Data are expressed as mean \pm SD. Bold number: $P<0.05$

Evaluation of intra-observer and inter-observer variability Intra-observer and inter-observer variability were examined in this study. We randomly selected 20 subjects from control and MHD groups for this evaluation. The intra-observer differences were compared between the two observations made by the same observer at 1 week. The inter-differences between observers were compared between two independent observers who were blinded to the grouping. The intra-observer and inter-observer variabilities were evaluated using the intra-class correlation coefficients (ICCs).

\section{Statistical analysis}

All statistical analyses were performed using SPSS 22.0 software (IBM SPSS, Statistics, Chicago, IL, USA). Measurement data are expressed as mean \pm standard deviation (SD), and significance was set at the level of $P<0.05$. The Kolmogorov-Smirnov's test was used to evaluate data normality. The independent sample $\mathrm{T}$ test was used to compare normally distributed data, while the non-parametric Mann-Whitney test was used to compare non-normal data. Pearson correlation analysis was used to examine the correlation between normally distributed variables, while Spearman correlation analysis was used for non-normal variables. The LS and CS values of the myocardium in each layer of the control subjects were defined as normal, and the LS and CS values of the myocardium in each layer of MHD patients were defined as abnormal. Subendocardial, mid-myocardial, and sub-epicardial LS and CS in MHD patients were analyzed using the receiver operating characteristic curve (ROC). The Yoden index was used to calculate the critical point of each strain value, specificity, and sensitivity. The criteria for ICCs were: "excellent" if $\geq 0.80$, "good" if $0.61-0.79$, "moderate" if $0.41-0.60$, and "poor" ICC $\leq 0.40$.

\section{Results}

Comparison of demographic and clinical characteristics between control and MHD groups

We first compared demographic and clinical data between control and MHD groups. As shown in Table 1, MHD patients had significantly higher SBP, DBP, and creatinine $(P<0.05)$ compared to the control group, but there were no significant differences with regard to age, gender, heart rate, and body mass index (BMI) between these two groups $(P>0.05)$. The primary cause of ESRD was glomerulonephritis (55.8\%), followed by diabetic nephropathy $(17.6 \%)$, hypertensive nephrosclerosis $(14.7 \%)$, and polycystic kidney (11.8\%) (Table 1).

\section{Comparison of traditional echocardiography parameters between control and MHD groups}

We next compared traditional echocardiography parameters between control and MHD groups. As shown in Table 2, LVEF was comparable between the groups $(P>$ $0.05)$, but MHD patients had significantly higher LVIDD, LVIDS, IVST, LVPWT, LAD, LVMI $(P<0.01)$ compared to the control subjects. These observations suggest that MHD patients had impaired cardiac function.

\section{Comparison of layer-specific strain parameters between control and MHD groups}

In both groups, LS and CS of the LV three-layer myocardium were consistent with the following pattern: sub-endocardial > mid-myocardium > subepicardial. Compared with the control group, the

Table 4 Comparison of horizontal layer-specific longitudinal strain between control and MHD groups

\begin{tabular}{|c|c|c|c|c|c|c|c|c|c|}
\hline \multirow{3}{*}{$\begin{array}{l}\text { Segmental LV } \\
\text { wall }\end{array}$} & \multicolumn{9}{|c|}{ Longitudinal strain (\%) } \\
\hline & \multicolumn{3}{|l|}{ Endocardial } & \multicolumn{3}{|l|}{ Middle } & \multicolumn{3}{|l|}{ Epicardial } \\
\hline & $\mathrm{MHD}$ & Normal & P-value & MHD & Normal & P-value & $\mathrm{MHD}$ & Normal & P-value \\
\hline basal segment & $-12.47 \pm 4.23$ & $-19.57 \pm 2.92$ & $<0.001$ & $-12.15 \pm 4.32$ & $-18.66 \pm 2.58$ & $<0.001$ & $-12.15 \pm 3.53$ & $-18.06 \pm 2.75$ & $<0.001$ \\
\hline middle segment & $-16.56 \pm 4.42$ & $-22.40 \pm 3.42$ & $<0.001$ & $-14.82 \pm 3.93$ & $-19.83 \pm 3.28$ & $<0.001$ & $-14.38 \pm 3.14$ & $-18.37 \pm 2.61$ & $<0.001$ \\
\hline apex segment & $-22.09 \pm 5.37$ & $-28.26 \pm 5.26$ & $<0.001$ & $-17.32 \pm 5.26$ & $-22.06 \pm 4.54$ & $<0.001$ & $-13.65 \pm 4.12$ & $-17.77 \pm 3.43$ & $<0.001$ \\
\hline
\end{tabular}

Data are expressed as mean \pm SD. Bold number: $P<0.05$ 

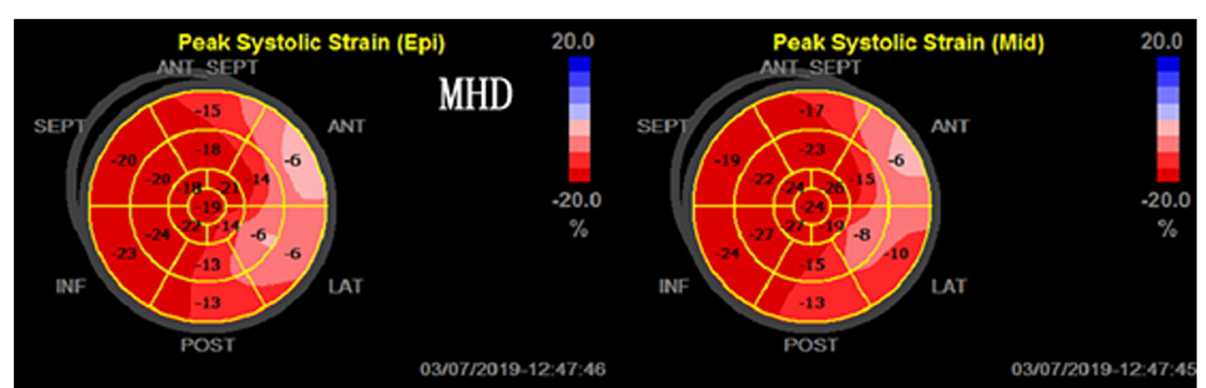

Peak Systolic Strain (Endo)

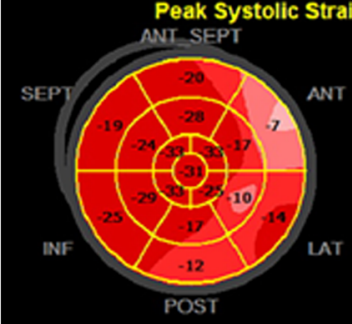

20.0
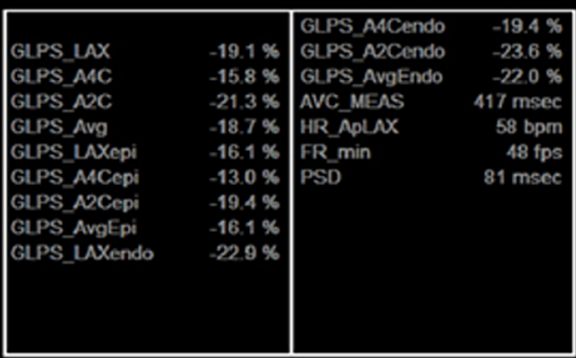

GLPS_A2Cendo

GLPS_AvgEndo

AVC_MEAS

HR_ApLAX
FR_min

PSD

$9.4 \%$

$2.9 \%$

3/07/2019-12:47:40

Peak Systolic Strain (Epi)

20.0

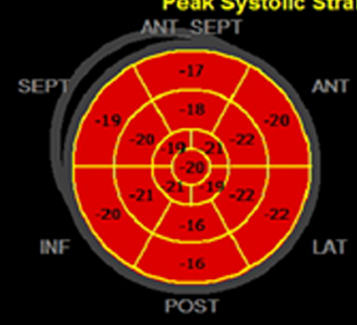

Normal

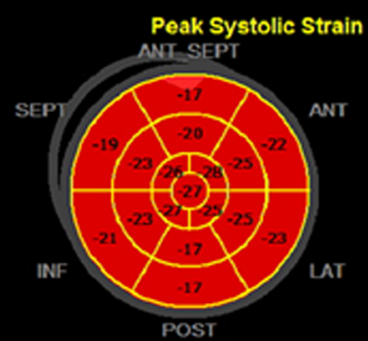

0

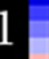

eak Systolic Strain (Endo)

03/07/2019-12:59:13
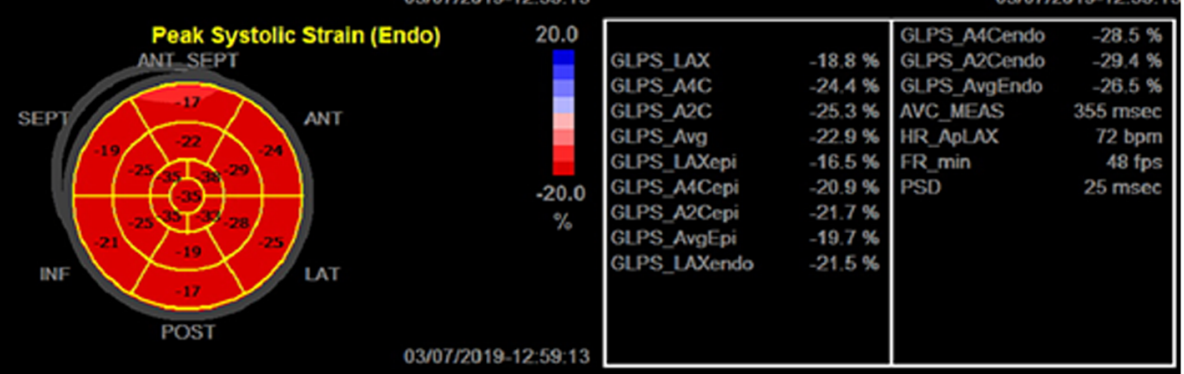

Fig. 1 Bull's eyes of the peak LS of the sub-endocardial, mid-myocardial, and sub-epicardial layers in MHD patients and normal subjects

MHD group had significantly lower LV GLS, GCS, and three-layer myocardial LS and CS $(P<0.01)$ (Table 3). MHD patients also had significantly lower LS of the LV three-layer at the basal, middle, and apical segments $(P<0.01)$ (Table 4, Fig. 1). In addition, with the exception of the sub-endocardium at the apical and middle segments, MHD patients had significantly lower CS of other LV layers compared to

Table 5 Comparison of horizontal layer-specific circumferential strain parameters between control and MHD patients

\begin{tabular}{|c|c|c|c|c|c|c|c|c|c|}
\hline \multirow{3}{*}{$\begin{array}{l}\text { Segmental LV } \\
\text { wall }\end{array}$} & \multicolumn{9}{|c|}{ Circumferential strain(\%) } \\
\hline & \multicolumn{3}{|c|}{ Sub-endocardial } & \multicolumn{3}{|l|}{ Mid-myocardial } & \multicolumn{3}{|l|}{ sub-epicardial } \\
\hline & $\mathrm{MHD}$ & Normal & $P$-value & MHD & Normal & $P$-value & $\mathrm{MHD}$ & Normal & $P$-value \\
\hline basal segment & $-23.88 \pm 5.99$ & $-27.46 \pm 5.37$ & 0.011 & $-15.15 \pm 3.60$ & $-19.09 \pm 4.18$ & $<0.001$ & $-9.47 \pm 2.62$ & $-13.11 \pm 3.64$ & $<0.001$ \\
\hline middle segment & $-23.79 \pm 5.40$ & $-26.00 \pm 3.80$ & 0.053 & $-14.44 \pm 3.52$ & $-17.97 \pm 3.26$ & $<0.001$ & $-8.56 \pm 3.15$ & $-12.43 \pm 3.17$ & $<0.001$ \\
\hline apex segment & $-26.41 \pm 7.23$ & $-28.74 \pm 5.25$ & 0.131 & $-16.41 \pm 3.49$ & $-19.77 \pm 4.03$ & $<0.001$ & $-9.56 \pm 3.41$ & $-11.54 \pm 3.39$ & 0.018 \\
\hline
\end{tabular}

Data are expressed as mean \pm SD. Bold number: $P<0.05$ 


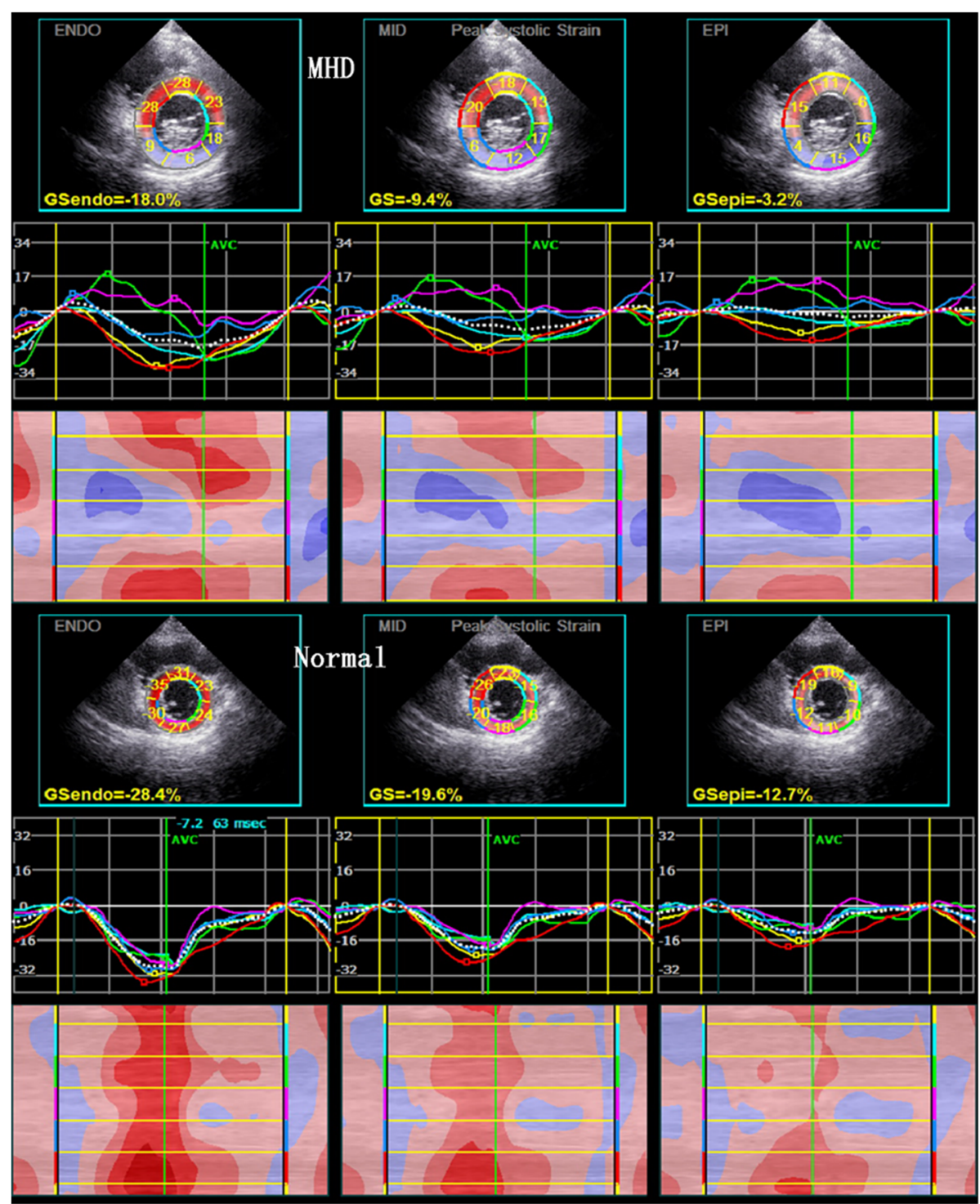

Fig. 2 Peak CS of the sub-endocardial, mid-myocardial, and sub-epicardial layers of LV in MHD patients and normal subjects

the control group $(P<0.05$ or $P<0.01)$ (Table 5, Fig. 2).

\section{Comparison of LV synchronous parameters between control and MHD groups}

In the control group, the color of the TTP bull's eye was uniformly green, while the color of the bull's eye in the MHD group was disordered, showing a mix of yellow and red, indicating that LV contraction was poorly synchronized (Fig. 3). The TTP of the LV 17 segments of MHD patients was significantly higher compared to the control group, and the peak time delay was observed in the majority of these segments (Table 6). In addition, MHD patients had significantly higher PSD compared to the control group $(P<0.01$, Table 7$)$. Taken together, these findings suggest that MHD patients had poorly synchronized LV systolic contraction, a sign of cardiac dysfunction.

\section{Correlation between PSD and GLS with three-layer LV myocardial LS}

We next examined the correlation between PSD and GLS with three-layer LV myocardial LS. As shown in Table 8 and Fig. 4, PSD was positively correlated with sub-endocardial and mid-myocardial LS and GLS (subendocardial, $\mathrm{r}=0.467, P=0.005$; mid-myocardial, $\mathrm{r}=$ $0.513, P=0.002$; GLS, $\mathrm{r}=0.463, P=0.006$ ). There was no significant correlation between PSD and sub-epicardial LS $(P=0.179)$. 

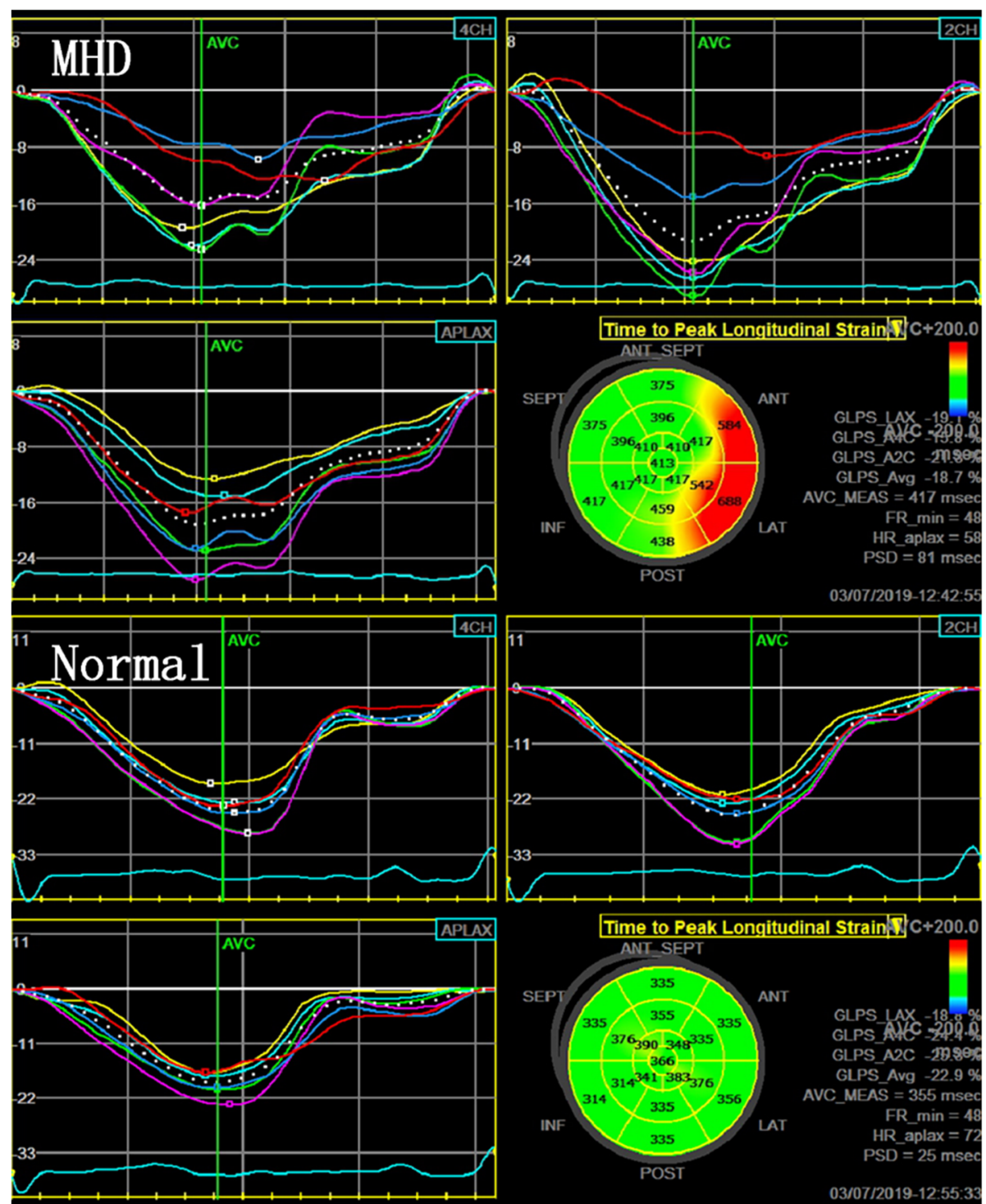

Fig. 3 Bull's eyes of the time to peak LS for the multiple LV segments and their PSD between MHD patients and normal subjects

ROC curve analysis of the accuracy of three-layer LV myocardial LS and CS in MHD patients

The AUC was analyzed to obtain the efficacy of threelayer LV myocardial LS and CS in the diagnosis of LV systolic dysfunction in MHD patients. The AUC values of sub-endocardial, mid-myocardial, and sub-epicardial LS myocardium in MHD patients were approximately $0.894,0.852$, and 0.870 , respectively, and the cutoff values were approximately $-21.15,-18.33 \%$, and $17.08 \%$, respectively. The sensitivity of sub-endocardial LS (85.3\%) was higher than that of sub-epicardial (82.4\%) and mid-myocardial LS (73.5\%). The specificity of LS of the sub-endocardial LS (82.9\%) was also higher than that of the mid-myocardial (80.0\%) and subepicardial LS (77.1\%). The AUC values of sub-epicardial, mid-myocardial, and sub-endocardial CS in MHD patients were approximately $0.852,0.837$, and 0.669 , respectively, and the cutoff values were $-11.65,-17.86 \%$, and $-24.33 \%$, respectively. The sensitivity of subepicardial CS (82.4\%) was higher than that of midmyocardial (73.5\%) and sub-endocardial CS (50.0\%). However, the specificity of mid-myocardial CS (82.9\%) was higher than that of sub-endocardial (80.0\%) and sub-epicardial CS (74.3\%) (Table 9, Fig. 5). Collectively, our findings indicate that sub-endocardial LS is an appropriate indicator of early stage cardiac dysfunction.

Intra-observer and inter-observer variability

We examined the intra-observer and inter-observer variability in our analysis. Twenty participants were randomly 
Table 6 Comparison of time to peak longitudinal strain of LV 17 segments between control and MHD groups

\begin{tabular}{llll}
\hline variable & \multicolumn{1}{l}{ TTP $(\mathrm{ms})$} & Normal & $P$-value \\
\cline { 2 - 4 } & MHD & $375 \pm 55$ & $\mathbf{0 . 0 3 2}$ \\
\hline bas ANT-SEPT & $415 \pm 93$ & $375 \pm 53$ & $\mathbf{0 . 0 0 6}$ \\
bas ANT & $424 \pm 85$ & $383 \pm 57$ & $<\mathbf{0 . 0 0 1}$ \\
bas LAT & $451 \pm 73$ & $379 \pm 51$ & $\mathbf{0 . 0 4 5}$ \\
bas POST & $405 \pm 54$ & $367 \pm 33$ & $\mathbf{0 . 0 1 7}$ \\
bas INF & $394 \pm 56$ & $376 \pm 52$ & 0.669 \\
bas SEPT & $389 \pm 71$ & $363 \pm 42$ & 0.499 \\
mid ANT-SEPT & $375 \pm 56$ & $360 \pm 39$ & $\mathbf{0 . 0 0 6}$ \\
mid ANT & $403 \pm 80$ & $366 \pm 38$ & $<\mathbf{0 . 0 0 1}$ \\
mid LAT & $431 \pm 67$ & $366 \pm 41$ & $\mathbf{0 . 0 0 2}$ \\
mid POST & $401 \pm 51$ & $360 \pm 30$ & $\mathbf{0 . 0 2 8}$ \\
mid INF & $381 \pm 48$ & $360 \pm 33$ & 0.094 \\
mid SEPT & $368 \pm 52$ & $365 \pm 33$ & $\mathbf{0 . 0 4 4}$ \\
ap ANT & $389 \pm 60$ & $369 \pm 33$ & 0.151 \\
ap LAT & $385 \pm 59$ & $358 \pm 28$ & $\mathbf{0 . 0 0 1}$ \\
ap INF & $397 \pm 61$ & $362 \pm 33$ & 0.372 \\
ap SEPT & $370 \pm 46$ & $364 \pm 28$ & $\mathbf{0 . 0 4 7}$ \\
ap AP & $383 \pm 46$ & $P<0.05$ & \\
\hline Da a & &
\end{tabular}

Data are expressed as mean \pm SD. Bold number: $P<0.05$

Bas basal segment; mid: middle segment; ap apex segment; ANT-SEPT anterior septum wall; ANT anterior wall; LAT lateral wall; POST posterior wall;

INF inferior wall; SEPT septum wall; TTP time to peak longitudinal strain

selected from the control and MHD groups. All observers were blinded to the subject type and measured the reproducibility of the three-layer LV myocardial LS, CS, GLS, GCS, and PSD. As shown in Table 10, our results suggest that our study generated reliable and consistent observations.

\section{Discussion}

Although MHD is the primary treatment for ESRD patients who are not candidates for kidney replacement, the mortality rate of MHD patients remains high and cardiovascular complications, such as heart failure and coronary heart disease, are the main factors associated with the high mortality of MHD patients [18]. Therefore,

Table 7 Comparison of LV PSD between control and MHD groups

\begin{tabular}{lll}
\hline Group & $\mathrm{n}$ & PSD \\
\hline MHD & 34 & $57.26 \pm 18.18$ \\
Normal & 35 & $29.97 \pm 8.31$ \\
$P$-value & & $<\mathbf{0 . 0 0 1}$ \\
\hline
\end{tabular}

Data are expressed as mean \pm SD. PSD: peak strain dispersion. Bold number: $P<0.05$
Table 8 Correlation between PSD and LV three-layer myocardial LS and GLS

\begin{tabular}{lclll}
\hline & \multicolumn{4}{l}{ Longitudinal strain (\%) } \\
\cline { 2 - 5 } & Endocardial & Middle & Epicardial & Global \\
\hline r-value & 0.467 & 0.513 & 0.236 & 0.463 \\
p-value & $\mathbf{0 . 0 0 5}$ & $\mathbf{0 . 0 0 2}$ & 0.179 & $\mathbf{0 . 0 0 6}$
\end{tabular}

Bold number: $P<0.05$

early detection of cardiac dysfunction in MHD patients has important clinical applications. In the present study, we used LST to evaluate the LS and CS of different LV myocardial layers of control and MHD patients and found that, despite the comparable LVEF between these two groups, MHD patients had altered LS and CS at different myocardial layers, higher TTP of 17 LV segments, and higher PSD compared to control subjects. Our findings suggest that LST holds value for early identifying LV dysfunction of MHD patients.

A previous study showed that hemodialysis did not significantly improve LV remodeling and systolic dysfunction in patients [19]. Consistent with that report, we first employed traditional echocardiography to assess the cardiac function of control subjects and MHD patients. We found that controls and MHD patients had a relatively comparable LVEF; however, MHD patients had significantly increased cardiac functional indexes, including LAD, LVIDD, LVIDS, IVSD, LVPWD, and LVMI. These findings suggest that ESRD patients exhibit LV remodeling with potential LV systolic dysfunction even after MHD treatment. Indeed, a previous study showed that ESRD patients on MHD had extensive pre- and post-loading factors such as sodium and water retention, anemia, malnutrition, valvular insufficiency, and hypertension [20]. The presence of a long-term arteriovenous fistula increased the pre-cardiac load in MHD patients [21]. To overcome increases in pre- and post-load, the LV develops physiological cardiac hypertrophy at an early stage to maintain normal cardiac output in MHD patients [22, 23].

Early studies have shown that the overall LS obtained by 2D-STE has become an objective and sensitive indicator for quantitative analysis of small changes in LV systolic function [24], which can be used to detect changes in regional myocardial blood supply earlier than LVEF [25]. LST based on 2D-STE technology is a new modality for evaluating wall motion. Animal experiments showed that LST can better assess the degree of myocardial infarctioninduced damage at an early stage [26]. Previous studies showed that the mid- and epi- myocardia of LV are sensitive to post-load changes [27-29], and that the endocardial myocardium is sensitive to changes in volumetric load $[27,30]$, indicating that different LV myocardial layers exhibit different responses to myocardial insults. Thus, 

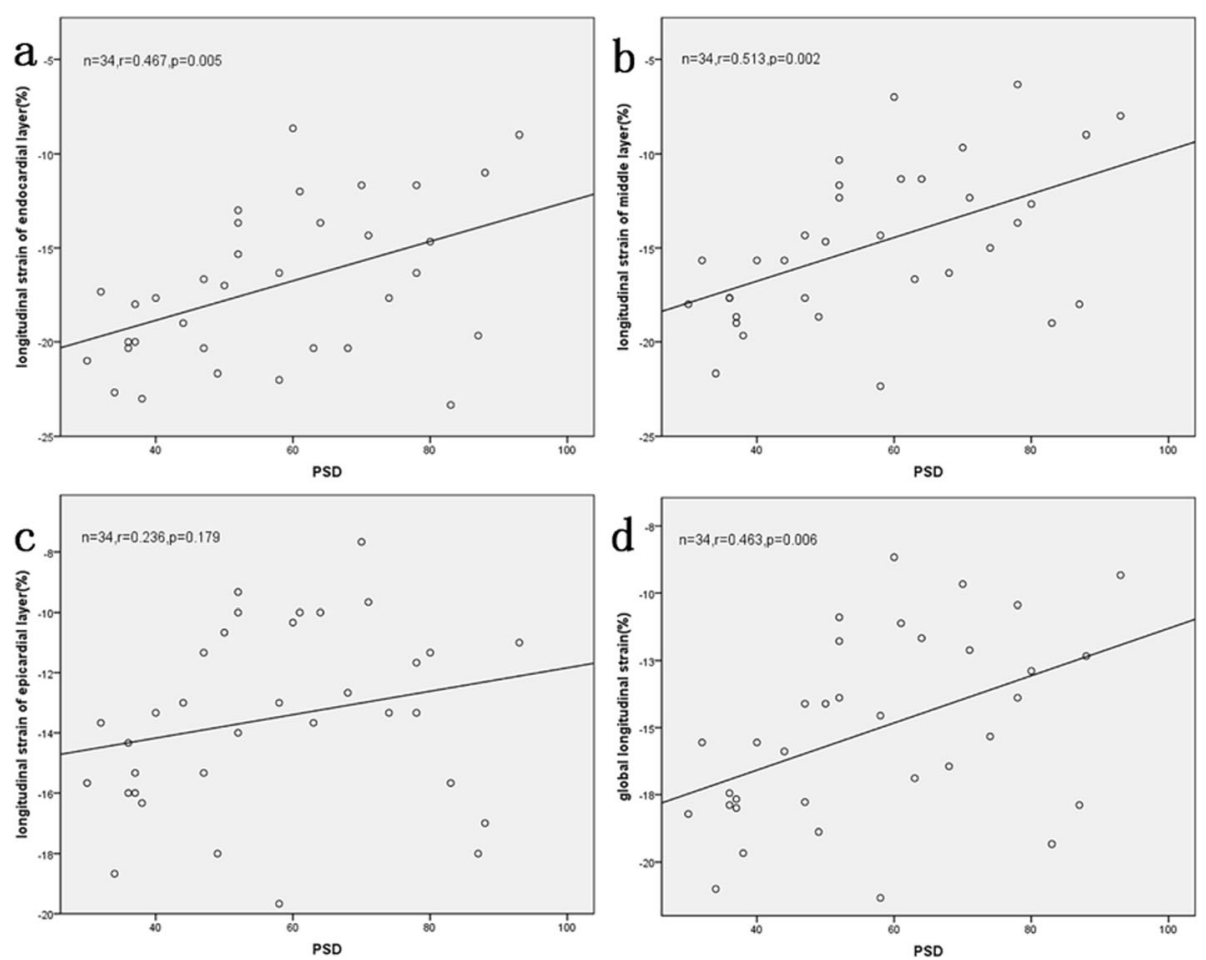

Fig. 4 Correlation between PSD and peak LS of the sub-endocardial (a), mid-myocardial (b) and sub-epicardial layers (c), and the GLS (d) in MHD patients

measuring layer-specific strain could be helpful to accurately assess the subtle changes in LV function during the progression of cardiac diseases. The present study used the layer-specific strain by $2 \mathrm{D}$ speckle tracking to appraise cardiac strain parameters of MHD patients, and revealed that MHD patients had significantly decreased myocardial LS of all layers compared to the control group. The impaired myocardial LS of all layers could be attributed to endothelial dysfunction and vascular injury [15], and to the observation that ESRD patients receiving MHD commonly present with anemia, secondary hyperparathyroidism, and arteriovenous fistula, all of which increase volume load and result in LV remodeling, hypertrophy, and fibrosis [31]. These pathological changes aggravate the progression of atherosclerosis and further heighten $\mathrm{LV}$ wall stress and stiffness [32], eventually leading to decreased endocardial LS [33]. As the disease progresses and dialysis time increases, myocardial fibrosis and LV hypertrophy is exacerbated, leading to a decrease in myocardial strain of each layer.

The cardiac myocardium can be divided into three layers: endocardial, middle, and epicardial. Approximately $75 \%$ of the endocardial and epicardial layers consist of longitudinal myocardium, while $25 \%$ of the middle layer consists of ring-shaped myocardium [34]. During myocardial contraction, the middle annular myocardium produces motion in the short axis direction. Compared with the control group, we found that MHD patients had comparable CS at the apical and the middle endocardial segments, indicating that LS was first

Table 9 ROC curve analysis of accuracy of LV three-layer myocardial LS and CS in MHD patients

\begin{tabular}{lllllll}
\hline Parameters & AUC & $95 \% \mathrm{Cl}$ & Cut-off Value (\%) & Sensitivity (\%) & Specificity(\%) & Youden index \\
\hline LS sub-endocardial & 0.894 & $0.796-0.955$ & -21.15 & 85.3 & 82.9 & 0.6521 \\
LS mid-myocardial & 0.852 & $0.746-0.926$ & -18.33 & 73.5 & 80.0 & 0.5353 \\
LS sub-epicardial & 0.870 & $0.767-0.939$ & -17.08 & 82.4 & 77.1 & 0.6244 \\
CS sub-endocardial & 0.669 & $0.545-0.778$ & -24.33 & 50.0 & 80.0 & 0.3000 \\
CS mid-myocardial & 0.837 & $0.728-0.915$ & -17.86 & 73.5 & 82.9 & 0.5639 \\
CS sub-epicardial & 0.852 & $0.746-0.926$ & -11.65 & 82.4 & 74.3 & 0.5664 \\
\hline
\end{tabular}

AUC: area under the curve; $95 \%$ Cl: 95\% confidence interval; LS: longitudinal strain; CS: circumferential strain 

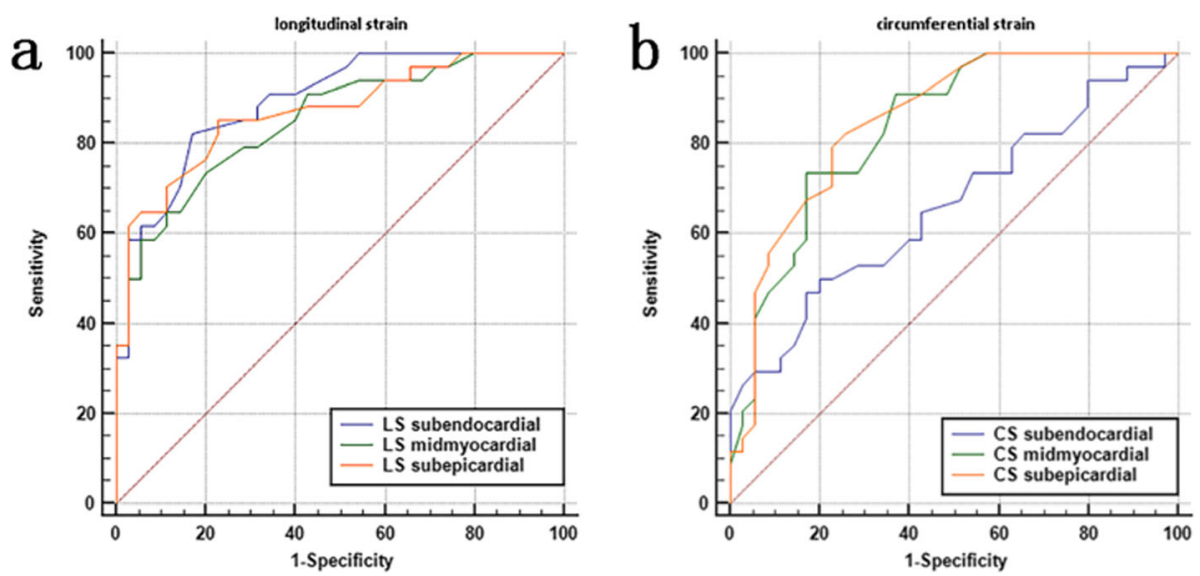

Fig. 5 ROC curve analysis for evaluating the sensitivity and specificity of the peak LS (a) and peak CS (b) of different myocardial layers in identifying LV dysfunction in MHD patients

impaired in MHD patients, while CS was only partially impaired at the early stage of cardiac impairment. Moreover, impaired CS was mainly observed in the middle and epicardial myocardium, which may be related to the fact that the middle myocardium has more ring-shaped myocardium. Since hemodialysis removes excess fluid from the body, the heart consequently increases LV contraction to maintain normal cardiac output and blood pressure [35]. However, MHD patients often have compromised cardiac function, and present with fluctuating blood pressure after hemodialysis. Since the mid- and epi-myocardium is sensitive to afterload [27-29], a large range of blood pressure fluctuation may underlie reduced CS in the mid- and epi-cardial myocardium of MHD patients.

LV TTP is the time from the R-wave of the electrocardiogram to the longitudinal peak strain of the LV 17 segments, and PSD measures the dispersion of the TTP of

Table 10 Evaluation of intra-observer and inter-observer variability

\begin{tabular}{llllll}
\hline & \multicolumn{2}{l}{ Intra-observer } & & \multicolumn{2}{l}{ Inter-observer } \\
\cline { 2 - 3 } & ICC & $95 \% \mathrm{Cl}$ & & ICC & $95 \% \mathrm{Cl}$ \\
\hline LS sub-endocardial & 0.90 & $0.76-0.96$ & & 0.87 & $0.69-0.94$ \\
LS mid-myocardial & 0.89 & $0.76-0.96$ & & 0.79 & $0.55-0.91$ \\
LS sub-epicardial & 0.87 & $0.70-0.95$ & & 0.88 & $0.72-0.95$ \\
GLS & 0.89 & $0.75-0.96$ & & 0.87 & $0.71-0.95$ \\
CS sub-endocardial & 0.89 & $0.74-0.95$ & & 0.87 & $0.71-0.93$ \\
CS mid-myocardial & 0.77 & $0.51-0.90$ & & 0.82 & $0.60-0.92$ \\
CS sub-epicardial & 0.86 & $0.69-0.94$ & & 0.80 & $0.57-0.92$ \\
GCS & 0.84 & $0.63-0.93$ & & 0.83 & $0.62-0.93$ \\
PSD & 0.96 & $0.90-0.98$ & & 0.95 & $0.88-0.98$ \\
\hline
\end{tabular}

ICC intra-class correlation coefficients; $95 \%$ Cl:95\% confidence interval; $L S$ longitudinal strain; GLS global longitudinal strain; CS circumferential strain; GCS global circumferential strain; PSD peak strain dispersion the LV 17 segments. Both TTP and PSD can be used to evaluate LV myocardial synchrony [36]. Compared with the control group, MHD patients had significantly delayed TTP and increased PSD, indicating decreased LV myocardial synchrony in MHD patients. This decreased LV synchrony could be attributed to increased sodium retention and high blood pressure. Indeed, some studies have shown that hypertension causes LV remodeling and alters myocardial electrophysiological properties, such as increased cardiomyocyte autorhythmicity and potential instability, myocardial electrophysiological conduction block, and myocardial excitation-contraction coupling dysregulation, all of which may lead to impaired LV systolic synchrony [37, 38]. Due to excessive volume overload, elevated peripheral blood pressure, and lack of ATP, LV contraction time is extended as a compensatory mechanism to insure adequate cardiac output [39]. Indeed, we found that PSD was positively correlated with GLS, which also indicates that the decrease in LV contraction in MHD patients is accompanied by a decrease in contractile synchrony. We also found that PSD was positively correlated with sub-endocardial and mid-myocardial GLS, but not with sub-epicardial GLS. This finding was probably due to the distribution of His bundle, left bundle branch, and Purkinje fiber mainly in sub-endocardium and mid-myocardium, thus rendering these myocardia more susceptible to the above factors [40].

Previously, Shi et al. [41] studied LV strain of young and middle-aged patients undergoing peritoneal dialysis, and found that sub-endocardial GLS was more sensitive to blood perfusion than the epicardium. Sun et al. [42] used LST technology to study LV function in ESRD patients, and reported that the curvature radius of the annular myocardial fiber was smaller than the curvature radius of the longitudinal myocardial fiber. Moreover, 
they found that the tension of the circumferential myocardial fiber was lower when deformed. Thus, they concluded that the LS of the myocardial fiber was more sensitive than CS. Leng et al. [43] also found that the decrease in LS occurred earlier than CS in response to external insults. In the present study, we analyzed the efficacy of three-layer strain parameters for predicting LV systolic function in MHD patients, and found that the AUC of the sub-endocardial LS was the largest with a cut-off value of $-21.15 \%$, indicating that the subendocardial LS was the best index to evaluate LV systolic function. The AUC of the mid- and sub-epicardial CS was greater than that of the sub-endocardial CS. Also, the AUC of the sub-endocardial LS was greater than that of the mid- and epi-cardial CS. Thus, our findings are in line with the previous reports.

\section{Limitations}

The limitations of this study should be acknowledged. First, our study was a single-centered study with a small sample size. Thus, we were not able to further divide MHD patients into subgroups. Second, the MHD patients in this study routinely took antihypertensive drugs, including calcium channel blockers and angiotensin converting enzyme inhibitors, and we did not rule out the possibility that the LV functional changes detected in MHD patients were already ameliorated by these treatments to some degree. Third, our study focused on LV longitudinal, circumferential strain, and synchrony, and did not include LV radial strain and torsion. Fourth, all MHD patients were assessed for coronary artery disease only by symptoms, electrocardiogram, myocardial enzyme profile, troponin, and echocardiography; they were not evaluated using angiography. Thus, our study did not rule out the possibility that some of these patients had subclinical coronary artery disease. Finally, the relationship between left ventricular load changes and left ventricular strain values revealed in MHD patients in this study needs to be further corroborated in the future.

\section{Conclusions}

We report here that, although MHD patients had relatively normal LVEF, they had significantly reduced LS and CS of sub-endocardial, mid-myocardial, and subepicardial layers as detected by the LST. MHD patients also had significantly increased PSD, suggesting impaired LV systolic synchrony. Therefore, LST can detect changes of three-layer strain, LV systolic function, and dys-synchrony in MHD patients with high sensitivity. Our findings provide direct evidence that LST may be used clinically to detect cardiac dysfunction at an early stage in MHD patients to guide cardiac treatment.

\begin{abstract}
Abbreviations
2D-STE: Two-dimensional speckle tracking echocardiography;

CS: Circumferential strain; ESRD: End stage renal disease; GCS: Global circumferential strain; GLS: Global longitudinal strain; IVST: Interventricular septal thickness at end-diastole; LAD: Left atrial diameter; LS: Longitudinal strain; LST: Layer-specific speckle tracking; LV: Left ventricular; LVEF: Left ventricular ejection fraction; LVIDD: Left ventricular internal diameter at enddiastole; LVIDS: Left ventricular internal diameter at end-systole; LVMI: Left ventricular mass index; LVPWT: Left ventricular posterior wall thickness at end-diastole; MHD: Maintenance hemodialysis; PSD: Peak strain dispersion; TTP: Time to peak longitudinal strain
\end{abstract}

\section{Acknowledgements}

Not applicable.

\section{Authors' contributions}

LC and YZN designed the study, collected, and analyzed echocardiography measurements. LC wrote the manuscript. YZN and FL revised the manuscript. FL and LC collected the clinical data. SD and HJ check the validity of data. LC and SXT performed the statistical analysis. All authors have read and approved the final manuscript.

\section{Funding}

None.

\section{Availability of data and materials}

The datasets generated and/or analyzed during the current study are available in the Open Science Framework repository,

https://mfr.osf.io/render?url=https://osf.io/bngwh/?action=download\%26 mode $=$ render

\section{Ethics approval and consent to participate}

This study complied with the Declaration of Helsinki and was reviewed and approved by the Human Subjects Committee of Changzhou No.2 People's Hospital approved this study. Written informed consent was obtained from the participant enrolled in this study.

\section{Consent for publication}

Not applicable.

\section{Competing interests}

The authors declare that they have no competing interests.

Received: 29 August 2019 Accepted: 30 December 2019

Published online: 09 January 2020

\section{References}

1. Shibiru T, Gudina EK, Habte B, Derbew A, Agonafer T. Survival patterns of patients on maintenance hemodialysis for end stage renal disease in Ethiopia: summary of 91 cases. BMC Nephrol. 2013;14(1):127.

2. Bleyer AJ, Tell GS, Evans GW, Ettinger WH Jr, Burkart JM. Survival of patients undergoing renal replacement therapy in one center with special emphasis on racial differences. Am J Kidney Dis. 1996;28:72-81.

3. Tonelli M, Karumanchi SA, Thadhani R. Epidemiology and mechanisms of uremia-related cardiovascular disease. Circ. 2016;13:518-36.

4. Ahmadmehrabi S, Tang WHW. Hemodialysis-induced cardiovascular disease. Semin Dial. 2018;31:258-67.

5. Shi Q, Zhu J, Feng S, Shen H, Chen J, Song K. Nonparallel progression of left ventricular structure and function in long-term peritoneal Dialysis patients. Cardiorenal Med. 2017;7:198-206.

6. Zoccali C. Prognostic value of echocardiographic indicators of left ventricular systolic function in asymptomatic Dialysis patients. J Am Soc Nephrol. 2004;15:1029-37.

7. Zoccali C, Benedetto FA, Mallamaci F, Tripepi G, Giacone G, Cataliotti A, et al. Prognostic impact of the indexation of left ventricular mass in patients undergoing dialysis. J Am Soc Nephrol. 2001;12(12):2768.

8. Longobardo L, Suma V, Jain R, Carerj S, Zito C, Zwicke DL, et al. Role of Two-Dimensional Speckle-Tracking Echocardiography Strain in the Assessment of Right Ventricular Systolic Function and Comparison with Conventional Parameters. J Am Soc Echocardiogr. 2017;30:937-46. 
9. Blessberger $H$, Binder T. NON-invasive imaging: two dimensional speckle tracking echocardiography: basic principles. Heart. 2010;96:716-22.

10. Stohr EJ, Shave RE, Baggish AL, Weiner RB. Left ventricular twist mechanics in the context of normal physiology and cardiovascular disease: a review of studies using speckle tracking echocardiography. Am J Physiol Heart Circ Physiol. 2016;311:H633-44.

11. Nagata $Y$, Wu VC, Otsuji Y. Takeuchi M Normal range of myocardial layer-specific strain using two-dimensional speckle tracking echocardiography. PLoS One. 2017;12:e0180584.

12. Shi J, Pan C, Kong D, Cheng L, Shu X. Left ventricular longitudinal and circumferential layer-specific myocardial strains and their determinants in healthy subjects. Echocardiography. 2016;33:510-8.

13. Huang J, Yan ZN, Fan L, Rui YF, Song XT. Left ventricular systolic function changes in hypertrophic cardiomyopathy patients detected by the strain of different myocardium layers and longitudinal rotation. BMC Cardiovasc Disord. 2017;17:214.

14. Hassanin N, Alkemary A. Early detection of subclinical uremic cardiomyopathy using two-dimensional speckle tracking echocardiography. Echocardiography. 2016:33:527-36.

15. Krishnasamy R, Hawley CM, Stanton T, Pascoe EM, Campbell KL, Rossi $M$, et al. Left ventricular global longitudinal strain is associated with cardiovascular risk factors and arterial stiffness in chronic kidney disease. BMC Nephrol. 2015;16:106.

16. Krishnasamy R, Isbel NM, Hawley CM, Pascoe EM, Burrage M, Leano R, et al. Left ventricular global longitudinal strain (GLS) is a superior predictor of all-cause and cardiovascular mortality when compared to ejection fraction in advanced chronic kidney disease. PLoS One. 2015; 10:e0127044.

17. Hämäläinen $H$, Hedman $M$, Laitinen $T$, Hedman $A$, Kivelä $A$, Laitinen $T$. Reference values for left ventricular systolic synchrony according to phase analysis of ECG-gated myocardial perfusion SPECT. Clin Physiol Funct Imaging. 2018;38:38-45.

18. Sun M, Kang Y, Cheng L, Pan C, Cao X, Yao H, et al. Global longitudinal strain is an independent predictor of cardiovascular events in patients with maintenance hemodialysis: a prospective study using three-dimensional speckle tracking echocardiography. Int J Cardiovasc Imaging. 2016;32:757-66.

19. Chen $R$, Wu $X$, Shen $L$, Wang $B, M a$ MM, Yang $Y$, et al. Left ventricular myocardial function in hemodialysis and nondialysis uremia patients: a three-dimensional speckle-tracking echocardiography study. PLoS One. 2014:9:e100265.

20. Ma W, Liu N, Tong M, Zhou H. Evaluation of left ventricular function in uremic patients by speckle tracking imaging. Cell Biochem Biophys. 2015;73:577-80.

21. Alkhouli M, Sandhu P, Boobes K, Hatahet K, Raza F, Boobes Y. Cardiac complications of arteriovenous fistulas in patients with end-stage renal disease. Nefrologia. 2015;35:234-45.

22. Foley RN, Curtis BM, Randell EW, Parfrey PS. Left ventricular hypertrophy in new hemodialysis patients without symptomatic cardiac disease. Clin J Am Soc Nephrol. 2010;5:805-13.

23. Lopez-Gomez JM, Verde E, Perez-Garcia R. Blood pressure, left ventricular hypertrophy and long-term prognosis in hemodialysis patients. Kidney Int Suppl. 1998;68:S92-8.

24. Kalam K, Otahal P, Marwick TH. Prognostic implications of global LV dysfunction: a systematic review and meta-analysis of global longitudinal strain and ejection fraction. Heart. 2014;100:1673-80

25. Adamo L, Perry A, Novak E, Makan M, Lindman BR, Mann DL. Abnormal Global Longitudinal Strain Predicts Future Deterioration of Left Ventricular Function in Heart Failure Patients With a Recovered Left Ventricular Ejection Fraction. Circ Heart Fail. 2017;10(6):e003788.

26. Bachner-Hinenzon N, Ertracht O, Malka A, Leitman M, Vered Z, Binah O, et al. Layer-specific strain analysis: investigation of regional deformations in a rat model of acute versus chronic myocardial infarction. Am J Physiol Heart Circ Physiol. 2012;303(5):H549-58.

27. Huang SJ, Orde S. From speckle tracking echocardiography to torsion: research tool today, clinical practice tomorrow. Curr Opin Crit Care. 2013;19(3):250-7.

28. Zhang $Q$, Fang F, Liang YJ, Xie JM, Wen YY, Yip GW, et al. A novel multi-layer approach of measuring myocardial strain and torsion by $2 \mathrm{D}$ speckle tracking imaging in normal subjects and patients with heart diseases. Int J Cardiol. 2011;147(1):32-7.
29. Duan F, Xie M, Wang X, Li Y, He L, Jiang L, et al. Preliminary clinical study of left ventricular myocardial strain in patients with non-ischemic dilated cardiomyopathy by three-dimensional speckle tracking imaging. Cardiovasc Ultrasound. 2012:10:8.

30. Imbalzano E, Zito C, Carerj S, Oreto G, Mandraffino G, Cusma-Piccione M, et al. Left ventricular function in hypertension: new insight by speckle tracking echocardiography. Echocardiography. 2011;28(6):649-57.

31. Nie Y, Zhang Z, Zou J, Liang Y, Cao X, Liu Z, et al. Hemodialysis-induced regional left ventricular systolic dysfunction. Hemodial Int. 2016;20(4):56472.

32. Widmer RJ, Lerman A. Endothelial dysfunction and cardiovascular disease. Glob Cardiol Sci Pract. 2014;2014(3):291-308.

33. Montgomery DE, Puthumana JJ, Fox JM, Ogunyankin KO. Global longitudinal strain aids the detection of non-obstructive coronary artery disease in the resting echocardiogram. Eur Heart J Cardiovasc Imaging. 2012;13:579-87.

34. Henein MY, Gibson DG. Normal long axis function. Heart. 1999:81:111-3.

35. A'Roch R, Gustafsson U, Johansson G, Poelaert J, Haney M. Left ventricular strain and peak systolic velocity: responses to controlled changes in load and contractility, explored in a porcine model. Cardiovasc Ultrasound. 2012; 10:22.

36. Dandel M, Lehmkuhl H, Knosalla C, Suramelashvili N, Hetzer R. Strain and strain rate imaging by echocardiography - basic concepts and clinical applicability. Curr Cardiol Rev. 2009;5(2):133-48.

37. Kim SA, Kim MN, Shim WJ, Park SM. Layer-specific dyssynchrony and its relationship to the change of left ventricular function in hypertensive patients. Heart Vessel. 2016;31(4):528-34.

38. Zoroufian A, Razmi T, Savandroomi Z, Tokaldany ML, Sadeghian H, Sahebjam M, et al. Correlation between systolic deformation and dyssynchrony indices and the grade of left ventricular hypertrophy in hypertensive patients with a preserved systolic ejection fraction undergoing coronary angiography, based on tissue Doppler imaging. J Ultrasound Med. 2014;33(1):119-28.

39. Ma Y, Zhang B, Zhang Y, Dong Y, Zhang R. Ultrasonic image analysis of longitudinal strain in uremic patients with preserved left ventricular ejection fraction. Biomed Eng Online. 2018;17(1):112.

40. Oh IY, Cha MJ, Lee TH, Seo JW, Oh S. Unsolved questions on the anatomy of the ventricular conduction system. Korean Circ J. 2018;48(12):1081-96.

41. Shi F, Feng S, Zhu J, Wu Y, Chen J. Left ventricular strain and Dyssynchrony in young and middle-aged peritoneal Dialysis patients and healthy controls: a case-matched study. Cardiorenal Med. 2018;8(4):271-84.

42. Sun M, Dong Y, Wang Y, Li G, Huang D. Assessment of the left ventricular function in patients with uremia using layer-specific 2-dimensional speckle tracking echocardiography. Med (Baltimore). 2019;98(9):e14656.

43. Leng Z, Li R, Li Y, Wang L, Wang Y, Yang Y. Myocardial layer-specific analysis in patients with heterozygous familial hypercholesterolemia using speckle tracking echocardiography. Echocardiography. 2017;34(3):390-6.

\section{Publisher's Note}

Springer Nature remains neutral with regard to jurisdictional claims in published maps and institutional affiliations.

Ready to submit your research? Choose BMC and benefit from:

- fast, convenient online submission

- thorough peer review by experienced researchers in your field

- rapid publication on acceptance

- support for research data, including large and complex data types

- gold Open Access which fosters wider collaboration and increased citations

- maximum visibility for your research: over $100 \mathrm{M}$ website views per year

At BMC, research is always in progress.

Learn more biomedcentral.com/submissions 